\title{
Construção e Validação de uma Escala de Competências Socioemocionais no Brasil
}

\author{
José Wilker de Lucena Macêdo ${ }^{1,2, *}$, Anielson Barbosa da Silva ${ }^{1}$
}

${ }^{1}$ Universidade Federal da Paraíba (UFPB), Brasil

${ }^{2}$ Instituto Nacional de Serviço Social (INSS), Brasil

Submissão: 19/12/2018

Primeira decisão editorial: 30/10/2019

Versão final: 10/11/2019

Aceite: $13 / 11 / 2019$

\section{Resumo}

O objetivo deste artigo é construir e validar uma escala de Competências Socioemocionais no Brasil. A prospecção dos itens foi realizada por meio da coleta de dados a partir de um grupo focal com gerentes de agências do Instituto Nacional do Seguro Social (INSS). Uma amostra de 424 indivíduos (220 mulheres e 204 homens) foi utilizada para avaliar a confiabilidade e a validade de 34 itens, mas o processo de análise exploratória preliminar dos dados possibilitou a exclusão de 9 itens. Os 25 itens da escala final foram agrupados em cinco competências, denominadas de consciência emocional, regulação emocional, consciência social, autocontrole emocional e criatividade emocional. A Escala de Competências Socioemocionais representa uma contribuição teórica nos campos da administração, da psicologia da educação e do trabalho porque foi desenvolvida e validada a partir de uma base teórico-empírica, considerando a realidade da cultura brasileira.

Palavras-chave: competência socioemocional, escala, emoções.

\section{Development and Validation of a Socioemotional Competences Scale in Brazil}

\section{Construcción y Validación de una Escala de Competencias Socioemocionales en Brasil}

Resumen

The purpose of this paper is the development and validation of a measure scale of Social-Emotional competences in Brazil. The prospecting of the items was performed by collecting data from a focus group with managers of the National Institute of Social Security (INSS) agencies. A sample of 424 individuals (220 women and 204 men) was used to evaluate the reliability and validity of the 34-Item of Social-Emotional scale, but the preliminary exploratory data analysis process that led the exclusion of 9 items. The 25-Item of the final Scale were grouped into five competences called emotional awareness, emotional regulation, social awareness, emotional self-control, and emotional creativity. The Social-Emotional competence scale represents a theoretical contribution in the field of Management, Education and Work Psychology because it was developed and validated from a theoretical-empirical basis considering the reality of the Brazilian culture.

Keywords: social-emotional competences, scale, emotions.
El objetivo de este artículo es construir y validar una escala de competencias socioemocionales en Brasil. La prospección de los ítems se realizó mediante la recopilación de datos de un grupo focal con gerentes de agencias del Instituto Nacional de Seguridad Social (INSS). Se usó una muestra de 424 individuos (220 mujeres y 204 hombres) para evaluar la confiabilidad y validez de 34 ítems, pero el proceso preliminar de análisis de datos exploratorios requirió la exclusión de 9 variables. Los 25 ítems finales de la escala se agruparon en cinco competencias que fueran denominadas de conciencia emocional, regulación emocional, conciencia social, autocontrol emocional y creatividad emocional. La escala de competencia socioemocional representa una contribución teórica en el campo de la Administración, de la Psicología de la Educación y del Trabajo porque se desarrolló y validó desde una base teórica y empírica considerando la realidad de la cultura brasileña.

Palabras-clave: competencias socioemocionales, escala, emociones. 
As competências socioemocionais (CSE) têm atraído a atenção de muitos pesquisadores desde o início dos anos 1990 (Ackley, 2016; Bar-On, 1997, 2016; Boyatzis \& Burkle, 1999; Goleman, 1995; Salovey \& Mayer, 1990; Schutte et al., 1998; Wong \& Law, 2002), cujos trabalhos apresentam pelo menos quatro objetivos principais: (1) apresentar um construto que relacione inteligência e emoção; (2) identificar as competências expressas pelo construto denominado inteligência emocional (IE) ou inteligência socioemocional (ISE); (3) descobrir se tais competências podem ser medidas; e (4) verificar o efeito dessas competências na vida das pessoas.

Existem vários instrumentos para mensurar CSE desenvolvidos nos Estados Unidos (Bar-On, 1997; Boyatzis, 2016, 2019; Mantz et al., 2016; Mayer, Caruso, \& Salovey, 2016), na Espanha (Pérez-Escoda, 2016; Pérez-Escoda, Filella, Alegre, \& Bisquerra, 2018; Sanchez-Garcia, Extremera, \& Fernandez-Berrocal, 2016; Zych, Ortega-Ruiz, Muñoz-Morales, \& Llorent, 2018), na China (Wong \& Law, 2002) e na América Latina (Mikulic, Crespi, \& Radusky, 2015).

Podemos identificar ao menos duas correntes teóricas associadas ao tema. A primeira corrente é filiada à perspectiva de Salovey e Mayer (1990), os quais definiram o construto IE como a percepção, o uso, a compreensão e a gestão das emoções em si e em outros. Esses autores publicaram o artigo seminal sobre o tema nos Estados Unidos (Salovey \& Mayer, 1990) e despertaram o interesse de várias outras pesquisas e publicações internacionais (Goleman, 1995; Schutte et al., 1998). Em um estudo mais recente, os autores passaram a compreender a IE como a capacidade de refletir sobre as emoções e de usá-las para melhorar o pensamento (Mayer et al., 2016), adotando instrumentos de aptidões aplicados por profissionais da área de psicologia para medir o que estes chamam de ISE (Teixeira \& Araújo, 2018).

Outra corrente teórica compreende o conceito de forma mais ampla, defendendo o uso do termo ISE para se referir às habilidades pessoais, emocionais e sociais que influenciam a capacidade do indivíduo de lidar com demandas, desafios e pressões diárias da vida (Bar-On, 1997, 2016; Boyatzis, 2016, 2019; Goleman, 1995). Em sua maioria, os autores dessa corrente utilizam instrumentos com versões do tipo autorrelato ou do tipo $360^{\circ}$ para medir o construto. Além disso, é muito mais comum o uso do termo CSE, pois este destaca as habilidades, os conhecimentos, as atitudes emocionais e as competências sociais, mas as duas últimas não são consideradas nos modelos de IE defendidos pela primeira corrente teórica apresentada neste artigo. Além das competências sociais, alguns autores também incluem traços de personalidade em sua definição (Bar-On, 1997; Goleman, 1995).

Pessoas com alto nível de CSE apresentam imagem adequada de si e de outros, maior facilidade para regular emoções nos níveis interpessoal e intrapessoal, para manter relacionamentos satisfatórios e para adotar comportamentos responsáveis em busca de bem-estar pessoal e social (Avci, Altindag, \& Yarbag, 2014; Løkke \& Madsen, 2014; Pérez-Escoda, 2016; Pérez-Escoda et al., 2018).

A partir da análise do conceito por vários autores, definimos as competências socioemocionais como a capacidade de tomar consciência, compreender, regular e expressar as emoções de forma apropriada para auxiliar na realização de tarefas, nos processos de aprendizagem, na resolução de problemas, na formação e na gestão de relacionamentos para se adaptar de forma mais efetiva às demandas complexas de crescimento e desenvolvimento ao longo da vida e aumentar o bem-estar pessoal e a qualidade das relações sociais (Boyatzis, 2016, 2019; Elias et al., 1997; Salovey \& Mayer, 1990; Pérez-Escoda, 2016).

O objetivo deste artigo é construir e validar uma escala de CSE no Brasil. Embora já existam instrumentos e pesquisas sobre CSE de gerentes (Ackley, 2016; Boyatzis, 2019), este trabalho inova por construir uma escala aplicável ao contexto brasileiro, desenvolvida a partir de uma base teórico-empírica e validada por especialistas e gerentes do serviço público federal, podendo ser utilizada para avaliar o nível de consciência emocional e social, de regulação e autocontrole emocional, além da criatividade emocional.

A opção de construir uma escala de CSE foi considerada mais adequada do que adaptar escalas existentes por três razões principais: (1) a preocupação com a perda semântica e /ou conceitual decorrente do processo de tradução dos itens (Cha, Kim, \& Erlen, 2007; Chapman \& Carter, 1979; Dias Júnior, 2016); (2) a dificuldade de acesso a alguns instrumentos de mensuração existentes que, em sua maioria, não estão disponíveis gratuitamente para os pesquisadores em geral; e (3) a relevância para a pesquisa na área da construção de um instrumento que considere a cultura dos respondentes, levando em conta a influência da cultura sobre a forma como expressamos e experimentamos emoções (Averill, 2005).

Como supracitado e referenciado, instrumentos de mensuração desenvolvidos ou adaptados considerando a cultura da amostra pesquisada são mais confiáveis. Contudo, após uma pesquisa por trabalhos que propusessem ou utilizassem escalas de CSE no Portal de Periódicos Capes (utilizando as expressões "scale" e "social and emotional competences" ou "escala" e "competências socioemocionais", buscando por assunto) e no Catálogo de Teses e Dissertações da Capes (utilizando as expressões "social and emotional competences" ou "competências socioemocionais"), não foram encontrados instrumentos de CSE validados ou adaptados ao contexto brasileiro.

Assim, este artigo está estruturado em quatro seções. A primeira aborda o referencial teórico sobre escalas de CSE e contextualiza a temática. Em seguida, na segunda seção, descreve-se a metodologia utilizada para o estudo empírico realizado com dados qualitativos e quantitativos. A terceira seção apresenta os resultados decorrentes das análises estatísticas realizadas e os discute à luz da literatura. Por fim, são feitas as considerações finais. Após as referências, apresentamos um apêndice com a Escala de CSE em sua versão final.

\section{Escalas de Competências Socioemocionais}

Mayer, DiPaolo e Salovey (1990) explicam que a IE envolve a avaliação e a expressão precisa de emoções em si e nos outros e a regulação emocional visando à melhoria da vida. $\mathrm{O}$ artigo empírico publicado por tais autores foi fundamental para o desenvolvimento de novas pesquisas com o objetivo de desenvolver um instrumento para mensurar habilidades emocionais, assim como o artigo teórico publicado por Salovey e Mayer (1990), o qual define a IE como um subconjunto da inteligência social (IS), ou seja, a habilidade de gerir pessoas para agir sabiamente nos relacionamentos humanos.

Os dois últimos artigos citados iniciam a discussão sobre a IE numa perspectiva teórica e contribuem para a formulação de outros questionamentos, tais como: se a IE é uma habilidade intelectual, ela pode ser medida e aprendida? Qual a melhor forma de mensurar tais habilidades? Qual o efeito das habilidades de IE no desempenho das pessoas ao longo da vida?

Alguns anos depois da publicação desses artigos, outros pesquisadores realizaram uma série de estudos (Schutte et al., 1998) com o objetivo de desenvolver e validar uma medida de IE com base no modelo proposto por Salovey e Mayer (1990). Esses autores afirmaram que os modelos de IE que surgiram paralelamente ao modelo de Salovey e Mayer (1990) não se contradizem, mas analisam o construto sob perspectivas diferentes da natureza da IE. Ademais, defendem que as inteligências intrapessoal (conhecer as próprias emoções) e interpessoal (conhecer as emoções e as intenções de outros) propostas por Gardner (2017) fundamentaram os modelos de IE que surgiram depois.

Utilizando o modelo de IE proposto por Salovey e Mayer (1990), Mayer et al. (2016) desenvolveram a Multifactor Emotio- 
nal Intelligence Scale (MEIS), uma escala de aptidão que mede a IE relacionando-a com o processamento informacional e a divide em quatro componentes: percepção emocional, facilitação emocional, entendimento emocional e administração emocional. Essa escala foi aperfeiçoada para o desenvolvimento do Mayer, Salovey \& Caruso Emotional Intelligence Test (MSCEIT).

Bar-On (1997) propôs o Inventário de Quociente Emocional (EQ-i), instrumento de mensuração de ISE do tipo autorrelato, com 133 itens com sentenças curtas e escalas de resposta de cinco pontos. Esses itens avaliam cinco dimensões de ISE, as quais são compostas por 15 competências socioemocionais: intrapessoal (autoestima, autoconsciência emocional, assertividade, independência e autoatualização), interpessoal (empatia, responsabilidade social e relacionamento interpessoal), gerenciamento do estresse (tolerância ao estresse e controle de impulsos), adaptabilidade (teste da realidade, flexibilidade e solução de problemas) e humor geral (otimismo e felicidade). Vários estudos empíricos utilizando o EQ-i foram realizados com o objetivo de comprovar que essas habilidades conseguem prever o comportamento e o desempenho humano (Bar-On, 1997).

Boyatzis (2016) apresenta um modelo de IE com 25 competências organizadas em cinco grupos. A partir do Self-Assessment Questionnaire (SAQ), instrumento de avaliação de competências de gerentes e líderes, os itens foram ajustados para as competências sociais e emocionais com foco em todas as ocupações e cenários da vida e o instrumento foi renomeado para Inventário de Competência Emocional (ECI), mais tarde substituído por Inventário de Competência Socioemocional (ESCI) (Boyatzis, 2016, 2019). Esse modelo de mensuração de ISE foi publicado após a validação contendo três grupos de competências: autoconhecimento, autoadministração e aptidões sociais.

Vale destacar que alguns pesquisadores de outros contextos culturais, como Europa (Pérez-Escoda, 2016), Oceania (Palmer \& Sough, 2001), América Latina (Mikulic et al., 2015) e Ásia (Wong \& Law, 2002), adaptaram tais modelos de IE e CSE ou desenvolveram novos modelos a partir destes para medir as CSE considerando aspectos culturais específicos.

Apesar de a literatura indicar algumas convergências entre os conceitos e as dimensões que compõem as escalas de IE e CSE, consideramos que as escalas de CSE são mais amplas, pois incorporam dimensões associadas às habilidades sociais, e mais adequadas quando consideramos contextos mais complexos da vida em sociedade, como o mundo do trabalho. Um exemplo dessa perspectiva é a evolução dos inventários propostos por Boyatzis (2016, 2019).

\section{Vantagens e Desvantagens das Escalas de CSE}

A IE tem sido estudada como o conjunto de habilidades de processamento emocional, com modelos que excluem algumas características de personalidade, tais como Salovey e Mayer (1990), e que a consideram um subconjunto da IS. Todavia, alguns modelos conhecidos como mistos incluem medidas de personalidade, como o modelo de Bar-On (1997) e Boyatzis (2016, 2019), denominados CSE ou ISE.

Vários instrumentos foram desenvolvidos para medir tal construto no modelo de autorrelato, de aptidões e habilidades. Muitos instrumentos de autorrelato também têm versões de avaliação $360^{\circ}$, que exigem a coleta de dados com uma pessoa e com seus pares, como o EQ-i e o ECI. Entre os instrumentos de medida de IE, existem aqueles aplicados ao mercado de trabalho (Wong \& Law, 2002). O ECI, embora tenha sido desenvolvido para se aplicar a todos os cenários da vida, possui competências importantes para medir a IE de gerentes e de pessoas no mercado de trabalho.

Em geral, as CSE são mensuradas de duas formas: por meio de instrumentos de autorrelato (Bar-On, 1997; Boyatzis, 2016; Mikulic et al., 2015; Pérez-Escoda, 2016; Wong \& Law, 2002) ou por meio de instrumentos de aptidão (Mayer et al., 1990, 2016). Os primeiros têm a vantagem de serem fáceis de aplicar, rápidos para responder e envolverem menos custos. Alguns deles também têm versões que incluem, além da resposta do indivíduo, a de seus pares, subordinados e/ou superiores, sendo chamados de versões do tipo $360^{\circ}$, tais como o MSCEIT, o ESCI e o EQ-i. As desvantagens desses instrumentos envolvem a subjetividade das respostas, ou seja, como não existe resposta certa ou errada, o avaliado pode simplesmente não responder de forma honesta por medo de se expor ou até mesmo por se enxergar de forma super/subvalorizada. Porém, em contextos relacionados ao local de trabalho e quando são aplicados em grandes amostras, tais instrumentos são mais utilizados e trazem bons resultados.

Os instrumentos de aptidão são mais caros e difíceis de aplicar. Alguns necessitam do auxílio de um profissional com formação específica para aplicação, são mais demorados para responder e têm muito itens (alguns mais de 400 itens, como é o caso do MEIS, e outros mais de 100 itens, como o MSCEIT). Esses instrumentos também podem apresentar problemas psicométricos. Por tais razões supracitadas, os instrumentos de autorrelato são mais utilizados em pesquisas realizadas com grandes amostras.

A Escala de Competências Socioemocionais desenvolvida nesta pesquisa é do tipo autorrelato, possui 25 itens, mede a frequência com que as CSE se expressam e foi construída considerando as especificidades da cultura brasileira, sendo validada com gestores públicos. A próxima seção descreve os procedimentos metodológicos utilizados para a construção e a validação da escala.

\section{Método}

\section{Participantes}

A pesquisa foi realizada em duas etapas: na primeira, de natureza qualitativa, houve a participação de 13 a 16 gestores que atuam em agências de um estado do Nordeste brasileiro; já a segunda, de natureza quantitativa, teve a participação de 424 gerentes de agências do Instituto Nacional do Seguro Social (INSS) (220 mulheres e 204 homens).

A análise dos dados dos respondentes da segunda etapa revelou que, em relação à região geográfica em que trabalham, 163 eram do Sudeste, 141 do Nordeste, 68 do Sul, 28 do Centro-Oeste e 24 do Norte. Já a idade dos respondentes variou entre 25 e 66 anos $(M=41,90$ anos; $D P=10,13)$. Cerca de $60 \%$ dos respondentes afirmou trabalhar entre 5 e 15 anos no INSS. Quanto ao tempo de trabalho como gestores, 36\% afirmaram atuar no cargo entre 1 e 5 anos, 27\% entre 6 e 10 anos, e $27 \%$ entre 11 e 15 anos. Quase 54\% da amostra possui renda bruta familiar entre 6 e 10 mil reais e, em relação ao nível educacional, cerca de $70 \%$ da amostra da segunda etapa tem ensino superior, enquanto os demais têm apenas ensino médio.

\section{Instrumentos}

Os itens da escala foram construídos a partir da revisão da literatura e da coleta de dados qualitativos por meio da realização de um grupo focal, em novembro de 2017, em três sessões. Foram convidadas a participar das sessões 25 pessoas, das quais 13 compareceram à primeira sessão e 16 compareceram às demais.

As sessões foram gravadas e transcritas, e os dados foram categorizados em dimensões de CSE com base na literatura. Os participantes discutiram em grupo sobre as CSE necessárias à sua ação gerencial (primeira e segunda sessões) e realizaram 
a validação de face e de conteúdo dos itens construídos ao final das duas primeiras sessões do grupo focal (terceira sessão).

Nas sessões de grupo focal, os participantes foram apresentados brevemente ao conceito de CSE, relacionado diretamente com o comportamento e a ação do indivíduo. Os participantes foram convidados a expressar quais e como mobilizam CSE em sua ação gerencial. A discussão foi estruturada em tópicos, escolhidos com base na literatura sobre o tema (Ackley, 2016): consciência emocional, consciência social, gerenciamento emocional e tomada de decisão responsável.

A partir dos resultados do grupo focal, a primeira versão da escala com 28 itens foi submetida à avaliação por 18 especialistas doutores nas áreas de administração e psicologia, dos quais 12 emitiram considerações sobre a escala ( 9 da área de administração e 3 de psicologia). Nessa etapa, foi solicitado aos especialistas que avaliassem qualitativamente os descritores quanto à clareza do enunciado e à adequação ao construto. Os dados coletados foram analisados com o objetivo de realizar a validação de face e de conteúdo dos descritores dos itens da escala.

A partir da revisão da literatura, da realização das sessões de grupo focal e da avaliação dos especialistas, 34 itens integraram a segunda versão da escala, que foi utilizada para a coleta de dados com os gerentes.

Os itens da segunda versão da escala foram organizados em um questionário, em ordem aleatória. Esse questionário foi estruturado com base em uma escala Likert variando de 1 a 6 , de acordo com a frequência que o indivíduo expressa cada competência (1 - nunca, 2 - quase nunca, 3 - às vezes, 4 - frequentemente, 5 - quase sempre, 6 - sempre).

\section{Procedimento de Coleta de Dados e Cuidados Éticos}

A aplicação do questionário foi realizada por meio de uma pesquisa do tipo Survey, no período de 2 a 20 de maio de 2018, utilizando o Google Forms. A pesquisa foi censitária, e o questionário foi enviado por e-mail para todos os 1.622 gerentes de agências do INSS. Os dados coletados foram inseridos em uma planilha do software SPSS (Statistical Package for the Social Sciences), versão Statistics Standard 22.0.

Em todas as etapas, a pesquisa adotou procedimentos éticos. Todos os participantes do grupo focal assinaram um termo de consentimento livre e esclarecido (TCLE), seguindo os preceitos da Resolução no 510/2016 do Conselho Nacional de Saúde. O consentimento dos participantes na pesquisa do tipo Survey foi realizado a partir da apresentação do TCLE na primeira página do questionário, na qual os participantes, para ter acesso às demais partes do instrumento, indicaram a concordância com as informações do termo.

\section{Procedimentos de Análise dos Dados}

A etapa da análise de dados do grupo focal foi qualitativa e seguiu os cinco passos propostos por Yin (2016): compilar, decompor, recompor (e arranjar), interpretar e concluir. A interpretação buscou seguir atributos de justeza, completude, precisão empírica, valor agregado e credibilidade. As expressões dos participantes revelaram que algumas competências mobilizadas eram características da cultura brasileira, por exemplo, foi dada ênfase à flexibilidade, à criatividade, à adaptabilidade, ao personalismo e à evitação de conflitos, características comuns entre os brasileiros (Budde, Scherer, Cassanego Junior, \& Vargas, 2019).

$\mathrm{Na}$ etapa quantitativa, os dados foram analisados inicialmente por meio da análise exploratória. Em seguida, foi realizada a análise fatorial exploratória (AFE) utilizando o método de componentes principais e rotação ortogonal Varimax, a análise de correlação entre os itens e a análise de confiabilidade dos itens. Inicialmente, foram realizados testes utilizando rotação ortogonal Varimax e oblíqua. Contudo, o primeiro tipo de rotação mostrou ser mais adequado do ponto de vista teórico, considerando o agrupamento dos itens por dimensões.

A exclusão dos itens foi realizada considerando de forma conjunta os resultados da AFE e das análises de correlação e confiabilidade. Considerou-se que o alfa de Cronbach acima de 0,7 , a correlação significativa entre itens da mesma dimensão entre 0,2 e 0,9 , e as cargas fatoriais e as comunalidades resultantes da AFE acima de 0,4 indicariam valores adequados, conforme proposto por Costa (2011). Os itens que não satisfizeram tais critérios foram excluídos. Por fim, foi realizada análise fatorial confirmatória (AFC) para validar a escala, de acordo com os critérios de validade convergente e discriminante, de confiabilidade e de adequação do modelo.

\section{Resultados}

A análise exploratória revelou ausência de valores perdidos (todos os respondentes responderam todas as questões do instrumento de pesquisa) e de valores extremos (não foi identificada resposta que fosse considerada muito alta ou muita bai$\mathrm{xa}$, induzindo os pesquisadores a descartá-la em decorrência de erro de preenchimento do instrumento de pesquisa). As variáveis apresentaram médias bem próximas das medianas. Porém, a análise dos histogramas das variáveis mostrou que elas não seguem uma distribuição normal. Após uma análise exploratória dos dados, decidiu-se não excluir nenhuma variável.

$\mathrm{Na}$ análise de correlação entre itens da mesma dimensão, apenas quatro não satisfizeram as condições propostas por Costa (2011). Foi extraída a primeira AFE com todas as variáveis utilizando o método de componentes principais e rotação Varimax com base em autovalores superiores a 1. Não se utilizou a rotação oblíqua, pois os componentes extraídos não se mostraram adequados ao modelo teórico utilizado. A extração foi realizada por meio do software SPSS. A matriz de componente rotativa resultou em sete fatores. Apenas dois itens apresentaram comunalidades e cargas fatoriais inferiores a 0,4 . Todos os itens apresentaram alfa de Cronbach acima de 0,7. Após análise conjunta das três análises (correlação entre itens de mesma dimensão, confiabilidade dos itens de mesma dimensão e AFE), oito itens foram excluídos.

Foi extraída a segunda AFE seguindo os mesmos procedimentos supracitados. Um item apresentou carga fatorial acima de 0,49 em mais de um fator. Consequentemente, ele foi excluído seguindo recomendações de Costa (2011).

Foi extraída a terceira e última AFE, seguindo os mesmos procedimentos anteriores. A extração resultou em cinco fatores. Todas as comunalidades e cargas fatoriais apresentaram valores maiores que 0,4 , exceto por um item que apresentou comunalidade igual a 0,375 . Porém, como o item apresenta relevância teórica, decidiu-se mantê-lo na escala. Os autovalores iniciais dos cinco fatores foram 9,07, 1,66, 1,30, 1,192 e 1,055, respectivamente. As cargas fatoriais e a variância explicada de cada fator são apresentadas na Tabela 1. Os cinco fatores explicam a variância de $60 \%$ da escala. Foi realizada AFC utilizando o software R (versão 3.3.1), pacote Lavaan, para a etapa de validação da escala.

\section{Validação da Escala}

A validação de face e conteúdo dos itens foi realizada tanto no grupo focal por gerentes de agência do INSS quanto na avaliação de especialistas conforme descrição apresentada na seção de metodologia.

Conforme a Tabela 2, a confiabilidade foi verificada por meio dos valores de ômega, todos maiores que 0,78 , e de alfa, todos 


\begin{tabular}{|c|c|c|c|c|c|}
\hline Itens por dimensão & F1 & F2 & F3 & F4 & F5 \\
\hline \multicolumn{6}{|l|}{ Consciência emocional (CE) } \\
\hline Entendo como os outros se sentem. & 0,72 & & & & \\
\hline Tenho relacionamentos marcados por atitudes de dar e receber afeto. & 0,70 & & & & \\
\hline Considero os sentimentos e as emoções dos outros antes de tomar uma decisão. & 0,67 & & & & \\
\hline Mantenho relacionamentos marcados pela confiança mútua. & 0,62 & & & & \\
\hline Considero as dificuldades das pessoas ao lidar com elas. & 0,57 & & & & \\
\hline Percebo as minhas emoções com facilidade. & 0,56 & & & & \\
\hline Compreendo como minhas emoções influenciam meu comportamento. & 0,46 & & & & \\
\hline \multicolumn{6}{|l|}{ Regulação emocional (RE) } \\
\hline Mantenho o desempenho satisfatório no trabalho/estudo mesmo diante de situações estressantes. & & 0,67 & & & \\
\hline Adapto-me facilmente a situações de mudança. & & 0,65 & & & \\
\hline Lido com várias demandas de trabalho/estudo sem perder o equilíbrio emocional. & & 0,59 & & & \\
\hline Busco a maior quantidade de informações antes de tomar uma decisão. & & 0,58 & & & \\
\hline Mantenho meu posicionamento em uma situação mesmo sob pressão. & & 0,57 & & & \\
\hline Defendo meus direitos de forma equilibrada mesmo sob pressão. & & 0,53 & & & \\
\hline \multicolumn{6}{|l|}{ Consciência social (CS) } \\
\hline Estou acessível quando minha equipe precisa de mim. & & & 0,72 & & \\
\hline Coopero quando trabalho em equipe. & & & 0,67 & & \\
\hline Assumo a responsabilidade pelas consequências de minhas decisões. & & & 0,65 & & \\
\hline Coloco-me no lugar do outro para ajudá-lo a lidar com suas dificuldades. & & & 0,60 & & \\
\hline \multicolumn{6}{|l|}{ Autocontrole emocional (AE) } \\
\hline Evito ter comportamentos explosivos diante de uma situação estressante. & & & & 0,79 & \\
\hline Controlo minhas palavras e ações mesmo sob pressão. & & & & 0,75 & \\
\hline Respeito os outros quando eles têm comportamentos diferentes dos meus. & & & & 0,62 & \\
\hline \multicolumn{6}{|l|}{ Criatividade emocional (CrE) } \\
\hline Encontro soluções criativas para os problemas que enfrento. & & & & & 0,65 \\
\hline Estimulo as pessoas a alcançarem um objetivo comum por meio do compromisso mútuo. & & & & & 0,63 \\
\hline Estabeleço parcerias com pessoas e organizações pensando no bem-estar social. & & & & & 0,55 \\
\hline Gerencio os conflitos de minha equipe no ambiente de trabalho/estudo de forma construtiva. & & & & & 0,55 \\
\hline Crio oportunidades para experimentar emoções positivas. & & & & & 0,40 \\
\hline \% Variância explicada & 36,3 & 6,6 & 5,2 & 4,7 & 4,2 \\
\hline
\end{tabular}

Nota. Fonte: elaborada pelos autores com base em extração obtida por meio do software SPSS (2018).

maiores que 0,75. De acordo com vários autores (Costa, 2011; Hair, Black, Babin, Anderson, \& Tatham, 2009; Marôco, 2010), valores de ômega acima de 0,7 apresentam bons níveis de confiabilidade.

A validade convergente foi verificada por meio da variância média extraída (avevar). A competência social e o autocontrole emocional apresentaram valores maiores que 0,5 , porém o mesmo não aconteceu com as demais competências. Portanto, para Consciência Social, Regulação Emocional e Criatividade Emocional não foi possível confirmar a validade convergente segundo esse critério, pois apenas valores acima de 0,5 são considerados adequados para validade convergente (Costa, 2011; Hair et al., 2009; Marôco, 2010).

Tabela 2

Medidas de validade convergente da escala

\begin{tabular}{cccccc}
\hline Medidas & CE & RE & CS & AE & CrE \\
\hline Alfa & 0,813 & 0,810 & 0,812 & 0,767 & 0,760 \\
Omega & 0,783 & 0,809 & 0,814 & 0,847 & 0,781 \\
Avevar & 0,376 & 0,415 & 0,523 & 0,580 & 0,401 \\
\hline
\end{tabular}

Nota. CE - Consciência Emocional, RE - Regulação Emocional, CS - Consciência Social, $\mathrm{AE}$ - Autocontrole Emocional e CrE - Criatividade Emocional. Fonte: elaborada pelos autores com base em extração obtida por meio do software R (2018).

Contudo, segundo o critério de cargas fatoriais padronizadas, todas precisam ser maiores que 0,5 e significativas. Tomando como referência esses parâmetros, a escala apresentou valores adequados para a validade convergente (Hair et al., 2009), conforme ilustra a Tabela 3.

A validade discriminante foi atendida seguindo o critério de Fornell e Larcker (1981), conforme se observa na Tabela 4, pois todos os valores das diagonais e em negrito (variâncias extraídas) são maiores que os valores que ficam abaixo delas na mesma coluna (variâncias compartilhadas entre as competências).

A adequação do modelo pode ser observada na Tabela 5 . O

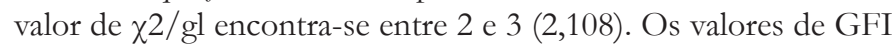
$(0,91)$, CFI $(0,93)$ e TLI $(0,92)$ são todos maiores que 0,9 . O RMSEA $(0,051)$ é maior que 0,5 e o $p$-valor $(0,000)$ é menor que 0,05 . Todos esses valores são considerados muito bons, e apenas o RMSEA é considerado aceitável (Costa, 2011; Hair et al., 2009; Marôco, 2010).

A escala validada em sua versão final foi apresentada na Tabela 3. As CSE foram classificadas em cinco dimensões: consciência emocional (sete itens), regulação emocional (seis itens), consciência social (quatro itens), autocontrole emocional (três itens) e criatividade emocional (cinco itens). As médias, desvios-padrão e correlações entre as CSE são apresentadas na Tabela 6.

\section{Discussão}

A principal contribuição deste artigo foi a construção e a validação de uma escala de CSE considerando o contexto brasileiro. Os critérios de confiabilidade, validade 
Consciência emocional (CE)

Tenho relacionamentos marcados por atitudes de dar e receber afeto.

Entendo como os outros se sentem.

Mantenho relacionamentos marcados pela confiança mútua.

Compreendo como minhas emoções influenciam meu comportamento.

Considero as dificuldades das pessoas ao lidar com elas.

Percebo as minhas emoções com facilidade.

Regulação emocional (RE)

Mantenho o desempenho satisfatório no trabalho/estudo mesmo diante de situações estressantes.

Adapto-me facilmente a situações de mudança.

Lido com várias demandas de trabalho/estudo sem perder o equilíbrio emocional.

Busco a maior quantidade de informações antes de tomar uma decisão.

Mantenho meu posicionamento em uma situação mesmo sob pressão.

Defendo meus direitos de forma equilibrada mesmo sob pressão.

Consciência social (CS)

Estou acessível quando minha equipe precisa de mim.

Coopero quando trabalho em equipe.

Coloco-me no lugar do outro para ajudá-lo a lidar com suas dificuldades

Assumo a responsabilidade pelas consequências de minhas decisões.

Autocontrole emocional (AE)

Respeito os outros quando eles têm comportamentos diferentes dos meus.

Controlo minhas palavras e ações mesmo sob pressão.

Evito ter comportamentos explosivos diante de uma situação estressante.

Criatividade emocional (CrE)

Encontro soluções criativas para os problemas que enfrento.

Estimulo as pessoas a alcançarem um objetivo comum por meio do compromisso mútuo.

Estabeleço parcerias com pessoas e organizações pensando no bem-estar social.

Gerencio os conflitos de minha equipe no ambiente de trabalho/estudo de forma construtiva.

Tabela 4

Variancias extraidas e compartilhadas

\begin{tabular}{cccccc}
\hline Dimensões & CE & RE & CS & AE & CrE \\
\hline CE & $\mathbf{0 , 8 5 1}$ & & & & \\
RE & 0,753 & $\mathbf{0 , 7 8 3}$ & & & \\
CS & 0,767 & 0,665 & $\mathbf{0 , 8 0 3}$ & & \\
AE & 0,597 & 0,627 & 0,699 & $\mathbf{0 , 8 7 2}$ & \\
CrE & 0,812 & 0,752 & 0,757 & 0,590 & $\mathbf{0 , 7 9 6}$ \\
\hline
\end{tabular}

Nota. CE - Consciência Emocional, RE - Regulação Emocional, CS - Consciência Social, AE - Autocontrole Emocional e CrE - Criatividade Emocional. Fonte: elaborada pelos autores com base em extração obtida por meio do software R (2018).

Tabela 5

Medidas finais da Escala de Competências Socioemocionais

\begin{tabular}{|c|c|c|c|c|c|c|c|}
\hline Qui-quadrado $\chi^{2}$ & $\begin{array}{c}g l \\
\text { (graus } \\
\text { lib.) }\end{array}$ & $\chi^{2 / g l}$ & $p$-valor & GFI & CFI & TLI & RMSEA \\
\hline 550,08 & 261 & 2,11 & 0 & 0,91 & 0,92 & 0,91 & 0,05 \\
\hline
\end{tabular}

Nota. p-valor - probabilidade de significância, GFI - Goodness of Fit Index, CFI - Comparative Fit Index, TLI - Tucker-Lewis index, RMSEA - Raiz do erro quadrático médio de aproximação. Fonte: elaborada pelos autores com base na extração obtida no software R (2018).

convergente e discriminante foram atendidos (Costa, 2011; Marôco, 2010). Também vale ressaltar que o modelo foi considerado adequado conforme recomendações de Hair et al. (2009).

Este trabalho se destaca por conta do seu ineditismo, uma vez
Tabela 6

Medidas das CSE identificadas

\begin{tabular}{cccccccc}
\multirow{2}{*}{ Competências } & Média & $\begin{array}{c}\text { Desvios- } \\
\text {-padrão }\end{array}$ & \multicolumn{4}{c}{ Correlação de Pearson } \\
\cline { 6 - 7 } & & CE & RE & CS & AE \\
RE & 32,54 & 5,01 & & & & \\
CS & 28,15 & 4,25 & $0,541^{*}$ & & & \\
AE & 21,65 & 2,54 & $0,611^{*}$ & $0,611^{*}$ & & \\
CrE & 14,29 & 2,49 & $0,495^{*}$ & $0,586^{*}$ & $0,491^{*}$ & \\
& 23,27 & 3,89 & $0,598^{*}$ & $0,605^{*}$ & $0,645^{*}$ & $0,476^{*}$
\end{tabular}

Nota. CE - Consciência Emocional, RE - Regulação Emocional, CS - Consciência Social, $\mathrm{AE}$ - Autocontrole Emocional e CrE - Criatividade Emocional. Fonte: elaborada pelos autores (2018)

que a construção e a validação da escala para mensurar CSE consideraram as especificidades da cultura brasileira, dado que todo o processo de delimitação dos itens foi realizado com a participação de gestores e especialistas brasileiros. Esse processo é muito significativo para o campo de pesquisa sobre as CSE no país.

Embora existam vários instrumentos validados internacionalmente para mensurar as CSE, nenhum deles foi construído ou adaptado levando em conta as particularidades da cultura brasileira. Isso se deve ao fato de que a forma de expressar emoções dos brasileiros é muito peculiar, e não se pode mensurar as CSE de um europeu ou de um norte-americano da mesma forma que se deseja mensurar as CSE de brasileiros. Portanto, a escala validada neste estudo tem relevante contribuição teórica e prática para a área da administração, da educação e da psicologia. 
Uma das vantagens da escala proposta é a sua quantidade de itens (25). Em estudos de autorrelato, muitas pessoas deixam de responder a um questionário ou o abandonam durante a indicação das respostas quando percebem que a quantidade de itens é elevada. Para a população desta pesquisa, um instrumento com muito itens seria um problema, devido ao ritmo intenso e ao elevado estresse no trabalho. O MSCEIT, por exemplo, é um tipo de instrumento de mensuração que envolve elevados custos em sua aplicação, pois precisa que alguém qualificado o aplique presencialmente (Boyatzis, 2016, 2019). O EQ-i possui versões com 133 itens (Bar-On, 1997), e o QDE-A (Cuestionario de Desarrollo Emocional de Adulto) com 48 itens (Pérez-Escoda, 2016). Portanto, a quantidade de itens da escala validada nesta pesquisa é uma vantagem em comparação às demais.

Das cinco dimensões da escala validada, quatro são comuns a outros modelos teóricos apresentados neste artigo: consciência emocional (Bar-On, 1997; Boyatzis, 2019; Elias et al., 1997; Mayer et al., 2016; Pérez-Escoda, 2016; Saarni, 2002), consciência social (Bar-On, 1997; Boyatzis, 2019; Pérez-Escoda, 2016), autocontrole emocional (Boyatzis, 2019) e regulação emocional (Pérez-Escoda, 2016; Salovey \& Mayer, 1990). A criatividade emocional é mensurada como um construto relacionado positivamente com a IE (Averill, 2005).

Comparou-se a confiabilidade da escala desenvolvida nesta pesquisa com outros instrumentos de mensuração. Os resultados demonstram que três dimensões apresentam maior confiabilidade quando comparadas a outros instrumentos de mensuração, e uma dimensão apresenta resultado um pouco menor. A confiabilidade da dimensão consciência social apresenta resultados melhores (alfa de Cronbach $=0,812$ ) quando comparada com outras escalas semelhantes, como o ESCI = 0,760 (Boyatzis, 2019), o EQ-i = 0,760 (Bar-On, 1997) e o QDE-A = 0,710 (Pérez-Escoda, 2016). A confiabilidade da dimensão consciência emocional (alfa de Cronbach $=0,812$ ) também apresenta resultados melhores quando comparada com outros trabalhos, como ECSI $=0,609$ (Boyatzis, 2019), EQ-i = 0,760 (Bar-On, 1997) e QDE-A = 0,700 (Pérez-Escoda, 2016). A dimensão de autocontrole emocional apresenta resultados um pouco menores (alfa de Cronbach $=0,767$ ) quando comparada à ECSI $(0,780)$. Por fim, a escala de regulação emocional apresenta confiabilidade maior (alfa de Cronbach = 0,810) quando comparada com o QDE-A = 0,800 (Pérez-Esco$\mathrm{da}, 2016)$. Quanto às cargas fatoriais obtidas por meio da AFC, são maiores quando comparadas com o EQ-i (Bar-On, 1997).

Outra vantagem desta escala é a amostra utilizada para validá-la. Os gerentes são uma amostra comumente pesquisada apenas para o ESCI entre as quatro escalas resumidas no parágrafo anterior (Boyatzis, 2019). Porém, por meio da atuação de gerentes, as organizações podem alcançar bons resultados coletivos. As CSE de gerentes precisam ser desenvolvidas como prioridade nas organizações, por isso uma escala que mede CSE de gerentes é de interesse para a gestão estratégica de pessoas e para o campo da psicologia do trabalho. As organizações que negligenciam o tema sofrem consequências sérias no desempenho das equipes e no clima organizacional como um todo.

Uma das dimensões propostas para a escala é a criatividade emocional, que foi indicada por Gondim et al. (2014) como um dos elementos que integram o construto CSE. Atualmente, o ritmo das mudanças no trabalho causadas pelas tecnologias da informação é muito intenso. Dessa forma, as pessoas lidam com situações complexas e diferentes todos os dias. Nesse contexto, é preciso ser criativo para resolver problemas, regular as próprias emoções e manter relacionamentos pessoais e parcerias no trabalho. Essa dimensão é extremamente relevante para as organizações porque pode contribuir na adaptação das equipes, na resiliência e na solução de problemas complexos.

Averill (2005) desenvolveu esse conceito ao longo dos anos, destacando o papel da criatividade ao lidar com as emoções na vida. $\mathrm{O}$ autor defende que a criatividade pode exercer o papel de estimular, inibir ou produzir emoções. Ao lidar com diversas situações no cotidiano, o indivíduo que busca de forma criativa experienciar emoções positivas é mais motivado, engajado e inovador. Contudo, vale destacar que Averill (2005) não compreende a criatividade emocional como uma CSE, mas a habilidade de expressar e experimentar um misto de emoções de forma autêntica, efetiva e inovadora, sendo tal habilidade relacionada à IE, assim como a criatividade está relacionada à inteligência. No entanto, Averill (2015) compreende que a criatividade emocional e as CSE têm habilidades em comum, mas também habilidades divergentes, ou seja, não são construtos totalmente diferentes. Este trabalho inova ao apresentar a criatividade emocional como uma CSE, convergindo teoricamente com a visão de Gondim et al. (2014).

\section{Considerações Finais}

As competências definidas na escala fornecem um panorama geral das competências essenciais para a ação gerencial em um contexto específico. Contudo, esses resultados podem ser aplicados a outros órgãos públicos no Brasil, subsidiando teoricamente os processos de gestão de pessoas relacionados ao desenvolvimento de competências e ao gerenciamento do desempenho no serviço público brasileiro. Por meio da avaliação dos níveis de CSE, pode-se criar programas de desenvolvimento de competências com o objetivo de melhorar o desempenho das pessoas no trabalho e, consequentemente, os resultados organizacionais. Tais programas precisam estar alinhados às diretrizes estratégicas organizacionais, sobretudo aos valores institucionais, e influenciar todos os subprocessos de gestão de pessoas.

Como sugestão de novos estudos, recomenda-se validar a escala em outros contextos profissionais, públicos ou privados, assim como verificar a relação entre as competências do instrumento com o desempenho acadêmico e no trabalho, a incidência de transtornos mentais e comportamentais, a efetividade gerencial, a renda das pessoas, as habilidades de inteligência geral, a satisfação no trabalho e a saúde e a qualidade de vida. Um estudo em desenvolvimento pelos autores pretende validar a escala com grupos multiprofissionais (gerentes, estudantes e docentes).

Uma das limitações deste trabalho envolve o tipo de instrumento utilizado para avaliar as CSE. Isso porque o instrumento de autorrelato pode produzir algum viés, uma vez que as pessoas podem não revelar seus pontos fracos ou podem se enxergar de forma distorcida, supervalorizando ou subestimando suas competências. Um possível instrumento complementar seria o tipo $360^{\circ}$, no qual o indivíduo se autoavalia e é avaliado por pares, superiores e subordinados. Porém, tal instrumento envolve uma grande mobilização de tempo e recursos financeiros, os quais não estavam disponíveis aos pesquisadores envolvidos neste trabalho. Os instrumentos de autorrelato são amplamente utilizados internacionalmente, produzem excelentes resultados e, além disso, são mais econômicos e mais fáceis de serem aplicados a amostras maiores (Bar-On, 1997; Boyatzis, 2019).

A validade convergente das competências definidas na escala foi considerada adequada, considerando as cargas fatoriais padronizadas das variáveis na AFC. Porém, possivelmente serão necessários alguns ajustes para que a variância média extraída das competências consciência emocional, equilíbrio emocional e criatividade emocional seja superior a 0,5 , conforme Hair et al. (2009). Esses ajustes poderão ser realizados em estudos futuros para validar a escala em outros contextos profissionais. 
A amostra utilizada neste trabalho corresponde aos indivíduos da população pesquisada que responderam ao instrumento e representa bem a população de gestores públicos do INSS. Contudo, pode não representar igualmente bem os demais gestores públicos brasileiros em outros tipos de organizações públicas, tais como autarquias, empresas públicas, fundações e sociedades de economia mista, bem como os gerentes do setor privado. Portanto, a amostra específica também pode ser considerada uma limitação da pesquisa, e a proposta de validar a escala com outros tipos de respondentes além de gestores - como alunos de ensino básico e superior, professores, profissionais da saúde e profissionais autônomos - também é um dos desafios futuros na popularização da escala no Brasil.

O Apêndice 1 apresenta as cinco competências da escala validada e seus respectivos conceitos. Os itens por dimensão estão disponíveis na Tabela 3. Essas competências não são expressas de forma exclusiva, pois seus conceitos não são excludentes. A expressão das CSE pelos indivíduos ocorre de forma fluida, por meio da mobilização de uma ou mais competências, não compartimentalizadas em caixas separadas. Portanto, por exemplo, ao expressar a consciência emocional, o indivíduo pode estar também expressando a criatividade e a regulação emocional ao mesmo tempo.

\section{Referências}

Ackley, D. (2016). Emotional intelligence: A practical review of models, measures, and applications. Consulting Psychology Journal: Practice and Research, 68(4), 269286. http://doi.org/10.1037/cpb0000070

Avci, S. B., Altindag, E., \& Yarbag, P. S. (2014). The effect of emotional intelligence on managerial involvement: An empirical study in Istanbul. International Journal of Research in Business and Social Science, 3(2), 86.

Averill, J. R. (2005). Emotions as mediators and as products of creative activity. Em J. Kaufman \& J. Baer (Eds.), Creativity across domains: Faces of the muse (pp. 225243). Mahwah, NJ: Erlbaum.

Averill, J. R. (2015). Emotion and anxiety: Sociocultural, biological, and psychological determinants. Em M. Zuckerman (Ed.), Emotions and Anxiety (PLE: Emotion, pp. 99-142). London: Psychology Press. https://doi. org/10.4324/9781315744643

Bar-On, R. (1997). The Emotional Quotient Inventory (EQ-I): Technical Manual. Toronto: Multi-Health Systems.

Bar-On, R. (2016). Beyond IQ and EQ. The Wiley Handbook of Personality Assessment, 104-118. https://doi.org/10.1002/9781119173489.ch8

Boyatzis, R. E., \& Burckle, M. (1999). Psychometric properties of the ECI: Technical Note. Boston: The Hay/McBer Group.

Boyatzis, R. E. (2016). Commentary on Ackley (2016): Updates on the ESCI as the behavioral level of emotional intelligence. Consulting Psychology Journal: Practice and Research, 68(4), 287-293. http://doi.org/10.1037/cpb0000074

Boyatzis, R. E. (2019). Emotional Intelligence and Its Measurement. Oxford Research Encyclopedia of Business and Management, 1-22. https://doi. org/10.1093/acrefore/9780190224851.013.159

Budde, J. S., Scherer, L. A., Cassanego Junior, P. V., \& Vargas, K. V. (2019). Cultura brasileira de gestão: um estudo em uma universidade pública do Rio Grande do Sul. Revista Gest@o.org, 17(1), 17-31. https://doi. org/10.21714/1679-18272019v17ed.p17-31

Cha, E. S., Kim, K. H., \& Erlen, J. A. (2007). Translation of scales in crosscultural research: issues and techniques. Journal of Advanced Nursing, 58(4), 386-395.

Chapman, D. W., \& Carter, J. F. (1979). Translation procedures for the crosscultural use of measurement instruments. Educational Evaluation and Policy Analysis, 1(3), 71-76.

Costa, F. D. (2011). Mensuração e desenvolvimento de escalas: Aplicações em administração. Rio de Janeiro: Ciência Moderna.

Dias Júnior, J. J. L. (2016). Adaptação e tradução de escalas de mensuração para o contexto brasileiro: Um método sistemático como alternativa a técnica back-translation. Métodos e Pesquisa em Administração, 1(2), 4-12. Recuperado de https://periodicos.ufpb.br/index.php/mepad/article/ view $/ 31880 / 16743$

Elias, M. J., Zins, J. E., Weissberg, R. P., Frey, K. S., Greenberg, M. T., Haynes, N. M., ... \& Shriver, T. P. (1997). Promoting social and emotional learning: Guidelines for educators. Virginia (USA): Ascd.

Fornell, C., \& Larcker, D. F. (1981). Evaluating structural equation models with unobservable variables and measurement error. Journal of Marketing Research, 18(1), 39-50. https://doi.org/10.1177/002224378101800104
Gardner, H. (2017). Taking a multiple intelligences (MI) perspective. Behavioral and Brain Sciences, 40, E203. https://doi.org/10.1017/S0140525X16001631

Goleman, D. P. (1995). Emotional intelligence: Why it can matter more than IQ for character, health and lifelong achievement. New York: Bantam Books.

Gondim, S. M. G., Morais, F. A., Brantes, C. A. A. (2014). Competências socioemocionais: fator-chave no desenvolvimento de competências para o trabalho. Revista Psicologia, Organizações e Trabalho, 14(4), 394- 406. Recuperado de http://pepsic.bvsalud.org/pdf/rpot/v14n4/v14n4a06.pdf

Hair, J. F., Black, W. C., Babin, B. J., Anderson, R. E., \& Tatham, R. L. (2009). Análise multivariada de dados. Porto Alegre: Bookman.

Løkke, A.-K., \& Madsen, H. (2014). Public sector managers and work stress. International Journal of Workplace Health Management, 7(2), 105-120. https:// doi.org/10.1108/IJWHM-03-2013-0009

Mantz, L. S., Bear, G. G., Yang, C., \& Harris, A. (2016). The Delaware socialemotional competency scale (DSECS-S): Evidence of validity and reliability. Child Indicators Research, 11(1), 137-157. https://doi.org/10.1007/s12187016-9427-6

Marôco, J. (2010). Análise de equações estruturais: fundamentos teóricos, software \& aplicações. Pêro Pinheiro: ReportNumber, Lda.

Mayer, J. D., Caruso, D. R., \& Salovey, P. (2016). The ability model of emotional intelligence: Principles and updates. Emotion Review, 8(4), 290-300. https:// doi.org/10.1177/1754073916639667

Mayer, J. D., DiPaolo, M., \& Salovey, P. (1990). Perceiving affective content in ambiguous visual stimuli: A component of emotional intelligence. Journal of Personality Assessment, 54(3-4), 772-781. https://doi.org/10.1080/00223891 .1990 .9674037

Mikulic, I. M., Crespi, M., \& Radusky, P. (2015). Construcción y Validación del Inventario de Competencias Socioemocionales para Adultos (ICSE). Interdisciplinaria: Revista de Psicología y Ciencias Afines, 32(2). https://doi. org/10.16888/interd.2015.32.2.7

Pérez-Escoda, N. P. (2016). Cuestionarios del GROP para la evaluación de la competencia emocional (CDE). Em J. L. Soler, L. Aparicio, O. Diaz, E. Escolano, \& M. A. Rodriguez (Eds.) Inteligencia Emocional y Bienestar II: reflexiones, experiencias profesionales e investigaciones (pp. 690-705). Ediciones Universidad de San Jorge.

Pérez-Escoda, N. P., Filella, G., Alegre, A., \& Bisquerra, R. (2018). Desarrollo de la competencia emocional de maestros y alumnos en contextos escolares. Electronic Journal of Research in Education Psychology, 10(28), 1183-1208. http:// doi.org/10.25115/ejrep.v10i28.1530

Saarni, C. (2002). Competência emocional: uma perspectiva evolutiva. Em R. BarOn \& J. D. A. Parker, Manual de inteligência emocional: Teoria e aplicações em casa, na escola e no trabalho. Porto Alegre: Artmed.

Salovey, P., \& Mayer, J. D. (1990). Emotional intelligence. Imagination, Cognition and Personality, 9(3), 185-211. https://doi.org/10.2190/dugg-p24e-52wk-6cdg

Sanchez-Garcia, M., Extremera, N., \& Fernandez-Berrocal, P. (2016). The factor structure and psychometric properties of the Spanish version of the MayerSalovey-Caruso Emotional Intelligence Test. Psychological Assessment, 28(11), 1404-1415. https://doi.org/10.1037/pas0000269

Schutte, N. S., Malouff, J. M., Hall, L. E., Haggerty, D. J., Cooper, J. T., Golden, C. J., \& Dornheim, L. (1998). Development and validation of a measure of emotional intelligence. Personality and Individual Differences, 25(2), 167-177. https://doi.org/10.1016/S0191-8869(98)00001-4

Teixeira, F. M., \& Araújo, A. M. (2018). Psychometric properties of the MayerSalovey-Caruso emotional intelligence test-MSCEIT V2.0: A systematic review of the literature. Revista Iberoamericana de Diagnostico y Evaluacion-E Avaliacao Psicologica, 3(48), 163-176. https://doi.org/10.21865/ridep48.3.14

Wong, C. S., \& Law, K. S. (2002). The effects of leader and follower emotional intelligence on performance and attitude. The Leadership Quarterly, 13(3), 243-274. https://doi.org/10.1016/s1048-9843(02)00099-1

Yin, R. K. (2016). Pesquisa qualitativa do início ao fim. Porto Alegre: Penso Editora.

Zych, I., Ortega-Ruiz, Ro., Muñoz-Morales, R., \& Llorent, V. J. (2018). Dimensions and psychometric properties of the social and emotional competencies questionnaire (SEC-Q) in youth and adolescents. Revista Latinoamericana de Psicologia, 50(2), 98-106. https://doi.org/10.14349/ rlp.2018.v50.n2.3

\section{Apêndice 1 - Dimensões e conceitos da escala validada (os itens por dimensão estão disponíveis na Tabela 3)}

\section{Consciência emocional (CE)}

Capacidade de reconhecer e compreender os sentimentos e as emoções de si próprio e dos outros, mantendo uma atitude compassiva, de confiança mútua, agindo com empatia nos relacionamentos e na tomada de decisão. 
Regulação emocional (RE)

Capacidade de manejar as emoções de forma adequada para manter um desempenho satisfatório em situações de estresse, em médio e longo prazos; adaptar-se a situações de mudança com flexibilidade e lidar com as situações de pressão.

\section{Consciência social (CS)}

Capacidade de cooperar quando trabalha em equipe, assumindo responsabilidades pelas suas ações, agindo com empatia e senso de colaboração ao reconhecer e entender as dificuldades do outro.

\section{Autocontrole emocional (AE)}

Capacidade de manter as próprias emoções sob controle, em curto prazo, diante de situações estressantes, assumindo uma atitude de respeito quando os comportamentos dos outros são diferentes dos meus.

\section{Criatividade emocional (CrE)}

Capacidade de usar as emoções de forma criativa para solucionar problemas, gerenciar conflitos de forma construtiva, criar oportunidades para experimentar emoções positivas e estabelecer parcerias em prol do interesse coletivo e do bem-estar social. 